\title{
Real-time sonoelastography of the patellar and quadriceps tendons: pattern description in profesional athletes and healthy volunteers.
}

\author{
Ayse Nur Sirin Ozcan ${ }^{1}$, Sinan Tan¹, Nuray Gulden Tangal', Saliha Curacı1, Savas Kudas², \\ Suleyman Bulent Bektaser ${ }^{3}$, Halil Arslan'
}

${ }^{1}$ Department of Radiology, ${ }^{2}$ Department of Sports Medicine, ${ }^{3}$ Department of Orthopedics and Traumatology, Ankara Atatürk Education and Research Hospital, Ankara, Turkey

\begin{abstract}
Aims: The comparison of elastographic features of quadriceps and patellar tendons in a group of professional athletes and healthy volunteers and the description of elasticity characteristics of these tendons. Material and methods: Thirtynine professional athletes (22 male, 17 female; mean age 18.5 years) and 35 healthy volunteers (21 male, 14 female; mean age 19 years) were included. They were divided into two groups by gender. Quadriceps tendon, patellar side of the patellar tendon, and tibial side of the patellar tendon elasticity patterns and strain ratios were investigated with real-time ultrasound elastography. The elasticity features of the dominant leg and non-dominant leg of athletes and volunteers legs were compared. In addition quadriceps and patellar tendons were compared separately for three distinct tendon locations. Results: There was no difference between the athletes and the healthy volunteers and also between the dominant leg and non-dominant leg of athletes. At tendon comparison, the quadriceps tendon was harder than the patellar tendon at both side and patellar side of patellar tendon was found to be stiffer than the tibial side of patellar tendon. Conclusions: Although biomechanical studies showed that tendon stiffness increased after long exercise, no significant difference was found between athletes' and healthy volunteers' tendon elasticity. These three tendon locations exhibit different elasticity features and the knowledge of the elasticity feature will be useful in assessing tendon pathologies.
\end{abstract}

Keywords: Real-time sonoelastography, quadriceps tendon, patellar tendon, musculoskeletal ultrasound

\section{Introduction}

Sonoelastography is a new ultrasonographic diagnostic technique evaluating the tissue elasticity based on differences in stiffness with pathological changes versus normal adjacent tissue $[1,2]$. There are several types of sonoelastography available and real-time ultrasound elastography (RTE) is one of the most widely used methods. In RTE, after compression with the transducer, the tissue that depicts more deformation is softer, and vice versa, less deformation corresponds to stiff tissue.

Received 01.02.2016 Accepted 14.04.2016

Med Ultrason

2016, Vol. 18, No 3, 299-304

Corresponding author: Ayse Nur Sirin Ozcan

Department of Radiology, Ankara Ataturk

Education and Research Hospital,

06800 Eskişehir Yolu 8.km No: 3 Bilkent

Ankara, Turkey

E-mail: aysenursirinozcan@gmail.com
Although it is known that pathological conditions change the elasticity of tissues, B- mod ultrasound cannot appreciate this modification and conventionally the elasticity is established just by manual palpation [2]. Instead of palpation, elastographic techniques are used for this purpose, offering qualitative and quantitative informations. RTE was used to study tissue elasticity alterations in different tissues such as thyroid, breast, lymph node, prostate, and musculoskeletal system [3-9].

Up to now, in musculoskeletal applications, RTE has been used especially for Achilles tendon imaging, the normal and abnormal Achilles tendon elasticity being studied [10-12]. RTE has been used for the assessment of patellar tendon elasticity in only one study, while no data are present in the literature concerning quadriceps elasticity.

The aim of our work was to describe patellar and quadriceps tendon RTE features in healthy volunteers and professional athletes. 


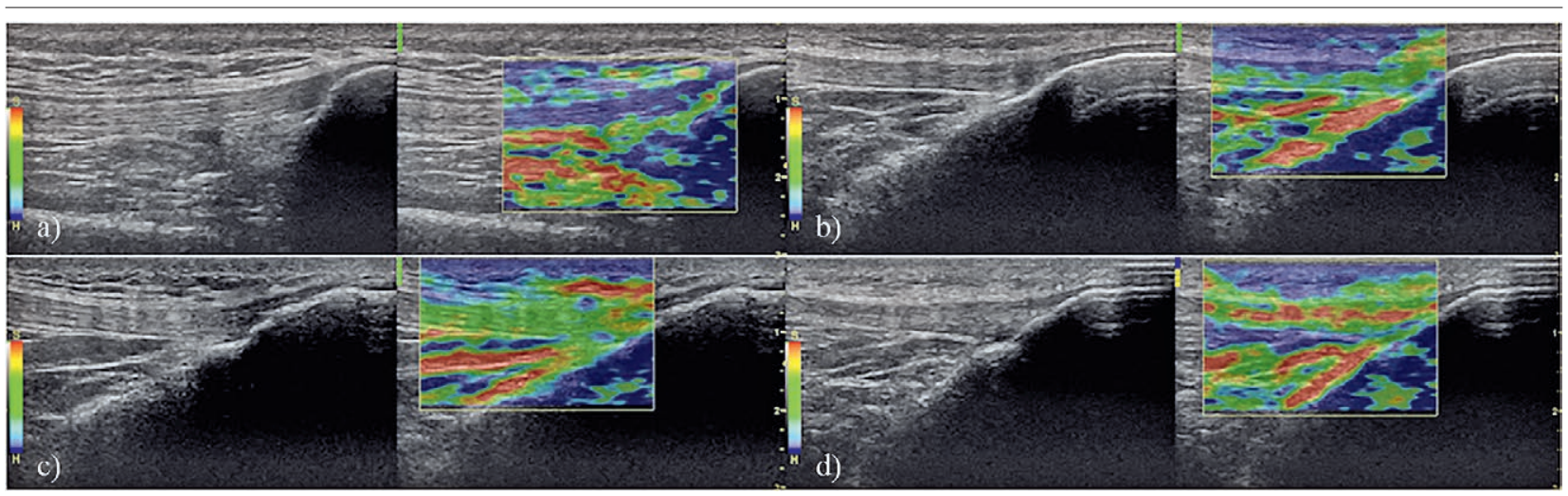

Fig 1. Elastographic pattern spectrum of patellar tendons: a) type 1 coloring of the quadriceps tendon; b) type 2 coloring on the patellar side of the patellar tendon; c) type 3 coloring on the patellar side of the patellar tendon; d) type 4 coloring on the tibial side of the patellar tendon.

\section{Material and methods}

\section{Study population}

The study was approved by the Ethical Committee at our institution and informed oral and written consent was obtained from all participants.

Quadriceps and patellar tendons of professional athletes and healthy volunteers were assessed in this study. Quadriceps and patellar tendon complaints or systemic inflammatory disorders that might have influenced our results, such as connective tissue, metabolic or endocrine diseases, or those taking steroids or estrogen medication were ruled out. During B-mod ultrasound, two patients were excluded from the study, one patient with intratendinous lipoma and one with calcification.

Thirty nine professional athletes (22 male, 17 female; mean age 18.5 years, range 18 -24 years) and 35 healthy volunteers (21 male, 14 female; mean age 19 years, range 18-23 years) were included in the study. A total of 148 leg and 444 tendon locations were assessed. The professional athletes' mean sports time was $7+/-0.3$ years.

\section{Examination protocol}

Before elastographic assessment, all 39 professional athlete and 35 healthy volunteer tendons were analyzed in grey scale ultrasound. Quadriceps and patellar tendons showed homogeneous and fibrillar echotexture in grey scale ultrasound for all subjects.

RTE was performed with a high frequency $6-15 \mathrm{MHz}$ linear probe, and a General Electrics Logiq E9 machine was used. All tendons were examined with the patient in the supine position and knee in a $20-30^{\circ}$ flexed position to deactivate the extensor mechanism and to avoid anisotropy due to the quadriceps tendon concave status. Bilateral quadriceps and patellar tendons were examined axially and longitudinally by a radiologist who was experienced in musculoskeletal ultrasound. RTE was performed in the

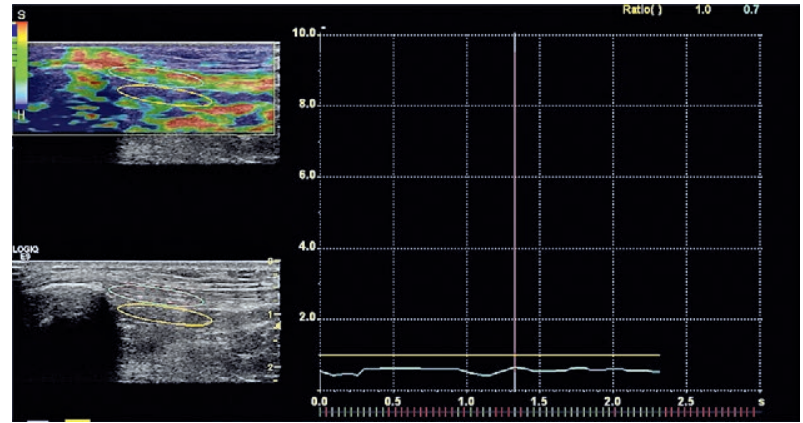

Fig 2. Strain ratio measurement from the patellar side of the patellar tendon with the elliptical ROI placed at the tendon and the fat pad beneath the tendon.

longitudinal plane and the transducer was placed perpendicular to the tendon. The RTE applied transducer position was observed from the B-mod window adjacent to the elastographic color window. The compression quality factor applied to the tendon was displayed on a bar scale from 1 to 7 and only images on the bar scale from 5 to 7 showing optimal compression were evaluated. Elastography measurements were also made from these images.

\section{Data analysis}

Measurements were performed from a single location at the distal end of the quadriceps tendon, and from two locations on the patellar and tibial side of the patellar tendon.

The elasticity pattern and strain ratio of tendons were investigated with RTE. According to the elasticity pattern, tendons were evaluated in 4 groups as blue (hardest tissue; type 1), blue-green (hard tissue; type 2), green (intermediate tissue; type 3), and yellow-red (soft tissue; type 4) (fig 1). With the strain ratio calculation, a semi-quantitative result was obtained comparing tendons with underlying fat pads: the one eliptical ROI was placed in the tendon and other in the suprapatellar fat pad (for the quadriceps tendon) and Hoffa's fat pad (for the patellar tendon) (fig 2). 
Table I. Mean strain ratios in tendons of professional athletes and healthy volunteers.

\begin{tabular}{llll}
\hline & Quadriceps tendon & Patellar tendon - patellar side & Patellar tendon - tibial side \\
\hline Male athlete DL & $1.73 \pm 0.32$ & $0.51 \pm 0.21$ & $0.41 \pm 0.13$ \\
Male athlete NDL & $1.76 \pm 0.5$ & $0.41 \pm 0.17$ & $0.34 \pm 0.17$ \\
Female athlete DL & $1.7 \pm 0.39$ & $0.62 \pm 0.23$ & $0.45 \pm 0.28$ \\
Female athlete NDL & $1.58 \pm 0.36$ & $0.59 \pm 0.25$ & $0.38 \pm 0.21$ \\
Male volunteer LL & $1.53 \pm 0.38$ & $0.55 \pm 0.22$ & $0.43 \pm 0.24$ \\
Female volunteer LL & $1.47 \pm 0.33$ & $0.60 \pm 0.17$ & $0.42 \pm 0.18$ \\
\hline
\end{tabular}

DL - Dominant leg, NDL - Non-dominant leg, LL - Left leg

Table II. Elasticity pattern distribution in all cases

\begin{tabular}{llll}
\hline & Quadriceps tendon & Patellar tendon - patellar side & Patellar tendon - tibial side \\
\hline Type 1 & $106(\% 71.6)$ & - & - \\
Type 2 & $41(\% 27.7)$ & $40(\% 27)$ & $5(\% 3.3)$ \\
Type 3 & $1(\% 0.67)$ & $76(\% 51.4)$ & $99(\% 60.1)$ \\
Type 4 & - & $32(\% 21.6)$ & $54(\% 36.5)$ \\
\hline
\end{tabular}

Professional athletes and healthy volunteers were divided into groups by gender, resulting in a total of four groups. For professional athletes, the commonly used extremity during sports activity was defined as the dominant side. To compare the professional athlete's dominant side with healthy tendon volunteers, the left side was selected since there was no dominance in the normal population. After noting all the values of the three locations (quadriceps tendon, patellar side of the patellar tendon, tibial side of the patellar tendon), the professional athletes' dominant side was compared with the non-dominant side and the professional athletes' dominant side was compared with the healthy volunteers' left extremity, individually for men and women, for elasticity pattern and strain ratio. All groups were combined and the three tendon location elasticity patterns and strain ratios were compared. Finally, the mean values were calculated individually for the three tendon locations for all cases.

\section{Statistical analysis}

Data analyses were performed using SPSS (Statistical Package for Social Sciences version 18.0 Chicago, IL, USA).

Professional athletes and healthy volunteers were divided into groups as male and female, a total of four groups. Statistical analysis was performed between these groups and also all groups were combined and the three tendon locations were compared.

Wilcoxon test, Mann-Whitney U test and Friedman test were used for statistical analysis of groups. MannWhitney U test was used to compare the professional athletes' dominant side with the healthy volunteers' left side tendon and Wilcoxon test was used for the comparison of the professional athletes' dominant side with the nondominant side.
After combining all groups, the Friedman test was used to determine the statistical relationship between the three tendon locations' elasticity pattern and strain ratio values. $\mathrm{p}<0.05$ was considered significant.

\section{Results}

No statistical differences were found for both men and women for the three tendon locations when comparing the dominant leg of athletes and the left leg of the control group or the dominant leg and non-dominant leg of athletes in terms of elasticity pattern and strain ratios, for both men and women $(\mathrm{p}>0.05)$. Professional athlete and healthy volunteer mean strain ratios are shown in table I.

Because of the lack of a significant difference, elasticity features of the professional athlete and healthy volunteer tendons for all groups were combined and the three locations' elasticity patterns and strain ratios were compared with each other. A significant difference was found between the quadriceps tendon and the patellar and tibial side of the patellar tendon of the same leg in terms of strain ratio (table II) and elasticity pattern $(\mathrm{p}<0.001)$. The quadriceps tendon was harder than the patellar tendon and the patellar side of the patellar tendon was harder than the tibial side $(p<0.001)$. Additionally, when we assessed the relationship between the tibial side and patellar side of the patellar tendon, the patellar side was found to be stiffer both in terms of elasticity pattern and strain ratio $(\mathrm{p}<0.001)$.

When all cases were assessed together, the elasticity pattern of the quadriceps tendon was calculated as $71.6 \%$ type 1 and $27.7 \%$ type 2 . The proximal patellar tendon was $27 \%$ type $2,51.4 \%$ type 3 , and $21.6 \%$ type 4 and 
the tibial side was $3.3 \%$ type $2,60.1 \%$ type 3 , and $36.5 \%$ type 4 (table II). When all cases were assessed together, strain ratios were $1.64 \pm 0.39$ in the quadriceps tendon, $0.55 \pm 0.22$ for the patellar side of the patellar tendon, and $0.40 \pm 0.20$ for the tibial side.

\section{Discussions}

The quadriceps and the patellar tendon are components of the knee extensor mechanism. In addition to contributing to knee function, knowledge about the biomechanical properties of the patellar tendon is important because it is commonly used as autograft material in the reconstruction of ligaments. Tendon strength and mechanical properties are affected by tendon dry mass, collagen content, and cross-linking [13-16]. Although the impact of exercise training on collagen content and cross-linking in human tendon tissue is unknown, animal data has shown increased collagen content with chronic training and biomechanical studies have revealed that tendon stiffness increases in tendons after long exercise $[17,18]$. This is the first study that has investigated the elasticity features of the patellar tendon in addition to the quadriceps tendon in athletes and healthy volunteers. With the definition of normal elasticity features of patellar and quadriceps tendons, we can constitute reference values and these values can give us additional information to grey scale US findings to use for the assessment of symptomatic and presymptomatic tendons.

In this study, professional athlete and healthy volunteer patellar tendon and quadriceps tendon elasticity patterns and strain ratios were put forward and differences between the two groups were explored as to whether exercise effects on tendon structure could be demonstrated with RTE. No differences were found between tendon elasticity features among professional athlete and healthy volunteer tendons or between the dominant and non-dominant knees of the healthy athletes as detected by Zhang et al [19]. When all tendons were assessed in the three distinct locations, the quadriceps tendon was harder than the patellar tendon and the patellar side of the patellar tendon was harder than the tibial side.

A tendon elasticity score was first developed for the Achilles tendon (on a 3-point scale) by De Zordo et al and afterwards different classifications were described by different researchers [10]. Ooi et al analyzed the patellar tendon as two groups - hard and soft - in their prospective study [20]. In the present study, we used four types of elasticity pattern, as detailed in Table 1 . These patterns, modified from the elastography pattern described by De Zordo et al constitute a new pattern to use for the quadriceps tendon and the patellar tendon [10]. Although biomechanical studies have shown that tendon stiffness increased in tendons such as tibialis posterior or Achilles after long exercise, in the present study no differentiation was found between tendon elasticity features between professional athletes' and healthy volunteer tendons [21]. In addition, the impact of physical activity on tendon elasticity biomechanical studies also point out that viscoelastic properties of the normal Achilles tendon varies with age and sex $[22,23]$. As our study, Drakonaki et al did not find any difference with age or sex [12]. On the other hand, Turan et al found that the Achilles tendon was remarkably stiffer in elderly subjects compared to young subjects in all parts of the tendon examination [24]. Despite biomechanical studies demonstrating different viscoelastic properties with physical activity, we could not quantify the difference with sonoelastography.

When all tendons were assessed at three distinct locations, the quadriceps tendon was harder than the patellar tendon and the patellar side of the patellar tendon was harder than the tibial side. In contrast to previously reported conclusions regarding tendon elasticity, the patellar tendon showed a more elastic pattern in our study. Our findings correlate with Porta et al results in which they found patellar tendon elasticity with RTE to be softer than other previously reported research on other tendons [25]. In addition, they found the patellar tendon patellar side to be green and violet and the tibial side to be mostly green, corresponding to our results showing the tibial side of the patellar tendon to be stiffer. Porta et al suggested that while a tendon connecting two bones appears highly elastic [25], in contrast to pateller tendon other tendons linking bones and muscles are stiffer. Maybe these tendons can be stiffer because of the elastic properties of the muscles they bind.

In addition to elasticity patterns, we performed a quantitative calculation (strain ratio) to evaluate tendon elasticity compared to the fat pad beneath the tendon. Tudisco et al use adjacent bone and Busilacchi et al used fat pad as the reference tissue to calculate strain ratio $[26,27]$. Fat pads were established as having a mosaic pattern with different colors, as described by Drakonaki et al [12]. They clarified this condition from stiffness differences in the boundaries between the fat lobules, fibrous septa, and vessels [12]. We found that the quadriceps tendon was harder than the patellar tendon and the patellar side of the patellar tendon was harder than the tibial side $(p<0.001)$. Additionally, when we assessed the relationship between the tibial side of the patellar tendon and the patellar side of the patellar tendon, the patellar side was found to be stiffer $(\mathrm{p}<0.001)$.

There are some limitations in this study. First of all, the professional athletes were not performing the same sport, 
with male professional athletes generally from football and females from volleyball. If we had a homogeneous athlete group comparison among athletes and healthy volunteers, this might have given us more accurate information.

Secondly, after athletes and healthy volunteers were divided into subgroups, the relatively small number of patients in each group may have led to limited statistical power.

The elastography technique, on account of the application of pressure to the probe, has general dependence on the individual operator, and therefore strain values may be affected by different degrees of manual compression. Another limitation was that interobserver and intraobserver variability were not assessed in our study. Although strain ratio calculation was a quantitative method, interobserver and intraobserver analysis assessment could enrich the elasticity pattern evaluation and show whether RTE results were dependent on the operator or not.

\section{Conclusion}

Sonoelastography is an appropriate technique for evaluation of quadriceps and patellar tendons. In our study, no significant difference was determined between athletes and healthy volunteers in terms of elasticity. When all tendons were assessed at three distinct locations we noticed that they exhibit different elasticity features. Knowledge of normal strain ratios and elasticity patterns in the healthy community will be useful in assessing pathologies of the tendon, especially when an accurate diagnostic approach to suspected tendonitis may be a diagnostic adjunct to ultrasound.

\section{Conflict of interest: none}

\section{References}

1. Ophir J, Cespedes I, Ponnekanti H, Yazdi Y, Li X. Elastography: a quantitative method for imaging the elasticity of biological tissues. Ultrason Imaging 1991; 13: 111-134.

2. Frey H. Real time elastography. A new ultrasound procedure for the reconstruction of tissue elasticity. Radiologe 2003; 43: 850-855.

3. Itoh A, Ueno E, Tohno E, et al. Breast disease: Clinical application of US elastography for diagnosis. Radiology 2006; 239: 341-350.

4. Miyanaga N, Akaza H, Yamakawa M, et al. Tissue elasticity imaging for diagnosis of prostate cancer: a preliminary report. Int J Urol 2006; 13: 1514-1518.

5. Dighe M, Bae U, Richardson ML, Dubinsky TJ, Minoshima S, Kim Y. Differential diagnosis of thyroid nodules with US elastography using carotid artery pulsation. Radiology 2008; 248: 662-669.
6. Thomas A, Kummel S, Gemeinhardt O, Fischer T. Realtime sonoelastography of the cervix: tissue elasticity of the normal and abnormal cervix. Acad Radiol 2007; 14: 193200.

7. Saftoiu A, Vilmann P, Hassan H, Gorunescu F. Analysis of endoscopic ultrasound elastography used for characterisation and differentiation of benign and malignant lymph nodes. Ultraschall Med 2006; 27: 535-542.

8. Janssen J, Schlorer E, Greiner L. EUS elastography of the pancreas: feasibility and pattern description of the normal pancreas, chronic pancreatitis, and focal pancreatic lesions. Gastrointest Endosc 2007; 65: 971-978.

9. Friedrich-Rust M, Ong MF, Herrmann E, et al. Real-time elastography for noninvasive assessment of liver fibrosis in chronic viral hepatitis. AJR Am J Roentgenol 2007; 188: 758-764.

10. De Zordo T, Fink C, Feuchtner GM, Smekal V, Reindl M, Klauser AS. Real-time sonoelastography findings in healthy Achilles tendons. AJR Am J Roentgenol 2009; 193: W134-W138.

11. Tan S, Kudaş S, Ozcan AS, et al. Real-time sonoelastography of the Achilles tendon: pattern description in healthy subjects and patients with surgically repaired complete ruptures. Skeletal Radiol 2012; 41: 1067-1072.

12. Drakonaki EE, Allen GM, Wilson DJ. Real-time ultrasound elastography of the normal Achilles tendon: reproducibility and pattern description Clin Radiol 2009; 64: 1196-1202.

13. Lemoine JK, Lee JD, Trappe TA. Impact of sex and chronic resistance training on human patellar tendon dry mass, collagen content, and collagen cross-linking. Am J Physiol Regul Integr Comp Physiol 2009; 296: R119-R124.

14. Kjaer M. Role of extracellular matrix in adaptation of tendon and skeletal muscle to mechanical loading. Physiol Rev 2004; 84: 649-698.

15. Last JA, Reiser RM. Collagen biosynthesis. Environ Health Perspect 1984; 55: 169-177.

16. Walker LB, Harris EH, Benedict JV. Stress-strain relationship in human cadaveric plantaris tendon: a preliminary study. Med Electron Biol Eng 1964; 2: 31-38.

17. Michna H. Morphometric analysis of loading-induced changes in collagen-fibril populations in young tendons. Cell Tissue Res 1984; 236: 465-470.

18. Woo SL, Ritter MA, Amiel D, et al. The biomechanical and biochemical properties of swine tendons-long term effects of exercise on the digital extensors. Connect Tissue Res 1980; 7: 177-183.

19. Zhang ZJ, Ng GY, Lee WC, Fu SN. Changes in morphological and elastic properties of patellar tendon in athletes with unilateral patellar tendinopathy and their relationships with pain and functional disability. PLoS One 2014; 9: e108337.

20. Ooi CC, Richards PJ, Maffulli N, et al. A soft patellar tendon on ultrasound elastography is associated with pain and functional deficit in volleyball players. J Sci Med Sport 2016; 19: 373-378.

21. Viidik A. Tensile strength properties of Achilles tendon systems in trained and untrained rabbits. Acta Orthop Scand 1969; 40: 261-272. 
22. Babic J, Lenarcic J. In vivo determination of triceps surae muscle-tendon complex viscoelastic properties. Eur J Appl Physiol 2004; 92: 477-484.

23. Kubo K, Kanehisa H, Fukunaga T. Gender differences in the viscoelastic properties of tendon structures. Eur J Appl Physiol 2003; 88: 520-526.

24. Turan A, Teber MA, Yakut ZI, Unlu HA, Hekimoglu B. Sonoelastographic assessment of the age-related changes of the Achilles tendon. Med Ultrason 2015; 17: 58-61.

25. Porta F, Damjanov N, Galluccio F, Iagnocco A, Matucc1Cerınic M. Ultrasound elastography is a reproducible and feasible tool for the evaluation of the patellar tendon in healthy subjects. Int J Rheum Dis 2014; 17: 762-766.

26. Tudisco C, Bisicchia S, Stefanini M, Antonicoli M, Masala $\mathrm{S}$, Simonetti G. Tendon quality in small unilateral supraspinatus tendon tears. Real-time sonoelastography correlates with clinical findings. Knee Surg Sports Traumatol Arthrosc 2015; 23: 393-398.

27. Busilacchi A, Olivieri M, Ulisse S, et al. Real-time sonoelastography as novel follow-up method in Achilles tendon surgery. Knee Surg Sports Traumatol Arthrosc 2016; 24 : 2124-2132. 